\title{
An approach to biologically important chromenes bearing P-S- heterocycles. Based on the chemistry of Lawesson's reagent
}

\author{
Maha D. Khidre* and Azza A. Kamel \\ Chemical Industries Division, National Research Centre, Dokki, D - 12622, Cairo, Egypt. \\ E-mail: mdkhedr@yahoo.com
}

\begin{abstract}
A series of chromenes bearing P-S-heterocycles, were prepared in reasonable yields from the reaction of 2,4-bis(4-methoxyphenyl)-1,3,2,4-dithiaphosphetane-2,4-disulfide (Lawesson's reagent, LR, 1) with a variety of substituted chromones. The antibacterial and /or antifungal activities for some of the new products obtained were evaluated.
\end{abstract}

Keywords: Lawesson's Reagent, substituted chromones, P-S-heterocycles, thiation, antibacterial activity, antifungal activity

\section{Introduction}

The activity of 2,4-bis(4-methoxyphenyl)-1,3,2,4-dithiaphosphetane-2,4-disulfide (Lawesson's reagent, LR, 1) as a thiating agent, has been studied in diverse classes of carbonyl compounds. ${ }^{1-3}$ At elevated temperatures, LR exists in equilibrium with the monomeric species $\mathbf{1 A}^{4}$ (Figure 1), which allows it to undergo $[2+4]$ cycloaddition with acyclic $\alpha$ - $\beta$-unsaturated ketones ${ }^{1,2,5}$ to give various heterocyclic compounds. Additionally, the ability of LR to produce 5- and 6-membered P-S-heterocycles from bifunctional systems has been previously discussed in the literature. ${ }^{6-8}$ Furthermore, the utility of LR for producing 4-membered P-S-heterocycles was previously reported by us. ${ }^{9}$ In continuation of this work, the present study has focused on the synthesis of bioactive P-S-heterocyclic systems. The methodology centered on the application of Lawesson's reagent with some chromone derivatives $2,7, \mathbf{1 1}$, and 15 . The structure-activity relationships of some of the isolated products are also briefly discussed. 


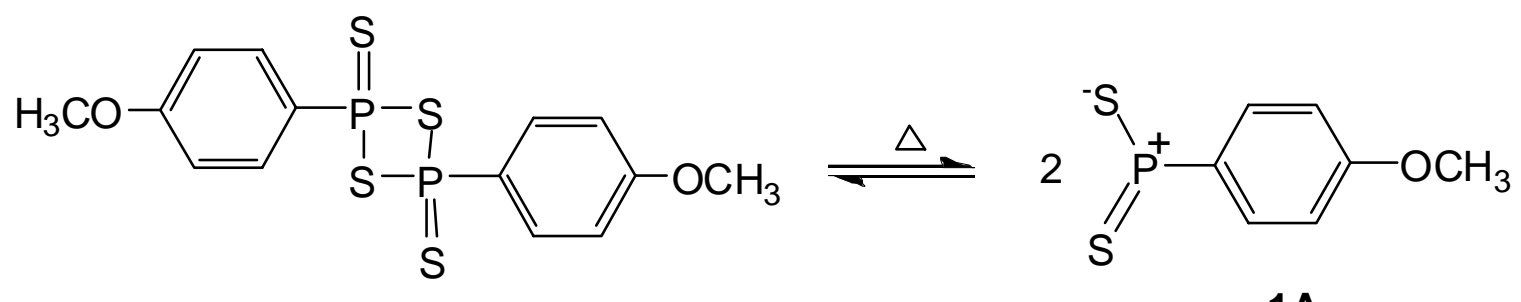

1

$1 \mathrm{~A}$

Figure 1

\section{Results and Discussion}

3-Formylchromone (2) was allowed to react with LR in boiling toluene to give a mixture of two products that could be separated by column chromatography. The first $(20 \%)$ is formulated as 4 thioxo-4-chromene-3-carbothialdehyde (3) based upon analytical and spectroscopic arguments. The second product $(60 \%)$, is formulated as 2-(4-methoxyphenyl)-5H-10aH$[1,3,2]$ oxathiaphosphinino[4,5-b]chromene-5-thione-2-sulfide (6) (Scheme 1).



2

$3(20 \%)$

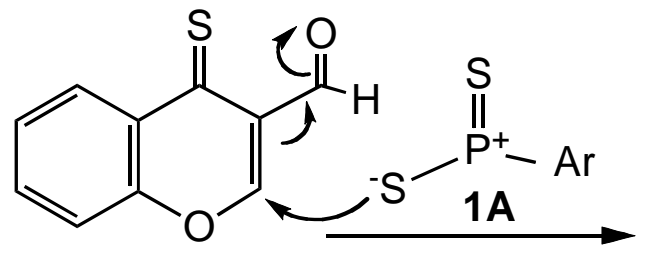

4<smiles>[O-]C=C1C(=S)c2ccccc2OC1S[P+]([Al-])=S</smiles>

5<smiles>[Al]P1(=S)OC=C2C(=S)c3ccccc3OC2S1</smiles>

$6(60 \%)$

$$
\mathrm{Ar}=\mathrm{C}_{6} \mathrm{H}_{4}-\mathrm{OCH}_{3}-p
$$

\section{Scheme 1}


The constitutions of the isolated products $\mathbf{3}$ and $\mathbf{6}$ were in accord with elemental analyses and spectroscopic properties. The structure of the oxathiaphosphinino chromene $\mathbf{6}$ was based on the following data: its ${ }^{31} \mathrm{P}-\mathrm{NMR}$ signal (in $\mathrm{CDCl}_{3}$, vs. $85 \% \mathrm{H}_{3} \mathrm{PO}_{4}$ ) was at $\delta 93.39$ which matches a cyclic structure. ${ }^{10,11}$ The IR spectrum of $\mathbf{6}$ revealed the absence of an absorption band around $1640 \mathrm{~cm}^{-1}$ corresponding to $(\mathrm{C}=\mathrm{O})$, instead, absorption bands at $1220(\mathrm{C}=\mathrm{S}), 1580,1600(\mathrm{C}=\mathrm{C}$, aromatic) $\mathrm{cm}^{-1}$ were present. Moreover, an absorption band at $650 \mathrm{~cm}^{-1}$ corresponding to $(\mathrm{P}=\mathrm{S})$ was present in the spectrum of $\mathbf{6}$. The ${ }^{1} \mathrm{H}$ NMR spectrum of 6 (in $\mathrm{CDCl}_{3}, \delta \mathrm{ppm}$ ) revealed the presence of signals at $3.85\left(\mathrm{~s}, 3 \mathrm{H}, \mathrm{OCH}_{3}\right), 7.4\left(\mathrm{~d},{ }^{3} J_{\mathrm{HP}}=11.3,1 \mathrm{H}, \mathrm{P}-\mathrm{S}-\mathrm{CH}\right)$. The AB system due to the four aromatic protons of the substituent atomatic ring appeared as two sets of doublet of doublets at 6.95 and 7.56 each with $J_{H H}=9 \mathrm{~Hz},{ }^{3} J_{H P}=11 \mathrm{~Hz}$, whereas the aromatic protons of the chromene ring appeared at 7.95, $8.7\left(2 \mathrm{~d}, 2 \mathrm{H}, J_{\mathrm{HH}}=7 \mathrm{~Hz}\right), 6.8,7.1(2 \mathrm{t}, 2 \mathrm{H},=\mathrm{CH}-\mathrm{CH}=), 8.8(\mathrm{~d}$, $\left.{ }^{3} J_{H P}=10.5,1 \mathrm{H}, \mathrm{P}-\mathrm{O}-\mathrm{CH}=\mathrm{C}\right)$. The ${ }^{13} \mathrm{C} \mathrm{NMR}$ showed signals at $55.30\left(\mathrm{OCH}_{3}\right), 84.71\left(\mathrm{~d},{ }^{2} J_{C P}=\right.$ 40.1, O-CH-S), 112.05 ( $\mathrm{S}=\mathrm{C}-\mathrm{C}=\mathrm{C}), 112.88,119.06,124.19,125.66,129.53,132.51,134.51$, $134.55,150.66,151.11,152.33,158.80\left(C=C\right.$, aromatic), $150.57\left(\mathrm{~d},{ }^{2} J_{C P}=38.9, \mathrm{C}=C-\mathrm{O}-\mathrm{P}=\mathrm{S}\right)$, $216.77(C=\mathrm{S})$.

The formation of compound 6 can be interpreted in terms of nucleophilic attack by the Sanion of the monomeric species 1A on the initially formed chromone thione intermediate $\mathbf{4}$ to give the transient dipolar structure 5 , followed by ring closure.

The reactions of 3-(aryliminomethyl)chromones 7a-d with LR were also studied. The isolated products are formulated as 3-\{(E)-[4-aryliminomethyl] $\}-4 H$-chromene-4-thione (8a-d) and 3-[3-aryl-2-(4-methoxyphenyl)-2-sulfido-1,3,2-thiazaphosphetidin-4-yl]-4H-chromene-4thione (10a-d) (Scheme 2). 
<smiles>[R]c1cccc(/N=C/c2coc3ccccc3c2=O)c1</smiles>

7a-d

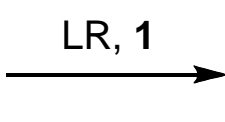<smiles>[R]c1ccc(N=Cc2coc3ccccc3c2=S)cc1</smiles>

8a-d ( 52\%)<smiles></smiles>

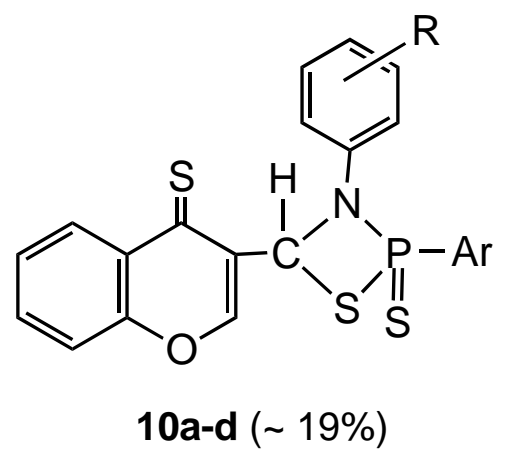
a, $\mathrm{R}=p-\mathrm{Cl}$
b, $\mathrm{R}=p-\mathrm{OCH}_{3}$
c, $\mathrm{R}=p-\mathrm{NH}_{2}$
d, $\mathrm{R}=\mathrm{O}-\mathrm{NH}_{2}$
$\mathrm{Ar}=\mathrm{C}_{6} \mathrm{H}_{4}-\mathrm{OCH}_{3}-p$

\section{Scheme 2}

The structure elucidation of 8a-d and 10a-d was based on their elemental analyses, molecular weight determinations (MS) and their spectroscopic data. For instance, the IR spectrum (in $\mathrm{KBr}$, $\mathrm{cm}^{-1}$ ) of 3-[3-(4-methoxyphenyl)-2-(4-methoxyphenyl)-2-sulfido-1,3,2-thiazaphosphetidin-4-yl]$4 \mathrm{H}$-chromene-4-thione (10b) showed the presence of absorption bands at $645 \mathrm{~cm}^{-1}$ corresponding to $(\mathrm{P}=\mathrm{S}), 1200 \mathrm{~cm}^{-1}(\mathrm{C}=\mathrm{S})$ instead of a lactone-carbonyl frequency at $1650 \mathrm{~cm}^{-1}$. The ${ }^{1} \mathrm{H}$ NMR spectrum of $\mathbf{1 0 b}\left(\mathrm{CDCl}_{3}, \delta \mathrm{ppm}\right)$ revealed the presence of a signal at $5.34(\mathrm{~d}, 1 \mathrm{H}$, $\left.{ }^{3} J_{H P}=12 \mathrm{~Hz}, \mathrm{~S}-\mathrm{CH}-\mathrm{N}\right)$, also two singlets due to the two $\left(\mathrm{OCH}_{3}\right)$ groups at 3.50 and $3.82 \mathrm{ppm}$. The aromatic protons $(13 \mathrm{H})$ appeared as a multiplet at $\delta_{\mathrm{H}}=6.45-8.32 \mathrm{ppm}$. The ${ }^{13} \mathrm{C} \mathrm{NMR}$ $\left(\mathrm{CDCl}_{3}, \delta \mathrm{ppm}\right)$ spectrum of 10b had signals at $\delta 54.59,55.21\left(2 \times \mathrm{OCH}_{3}\right), 67.57\left(\mathrm{~d},{ }^{2} J_{C P}=42\right.$, P-S-CH), 115.35, 117.17, 118.33, 119.45, 122.56, 124.92, 129.84, 132.55, 134.64, 135.22, $139.99,149.85,153.40,155.55,158.34,160.95$ (aromatic carbon atoms), 205.67( $C=\mathrm{S})$.

According to Scheme 2, the formation of 10a-d is believed to occur via nucleophilic attack of the monomeric species $\mathbf{1 A}$ on compound $\mathbf{8}$ to give intermediate $\mathbf{9}$, followed by ring closure to afford the thiazaphosphetidin chromene $\mathbf{1 0 .}$

In the same sense, [(4-oxo-4H-chromen-3-yl)methylene]malononitrile 11 reacted with LR to give a mixture of two products which could be separated by column chromatography. The first product $(65 \%)$ is formulated as (2E)-2-cyano-3-(4-thioxo-4-chromen-3-yl)prop-2-ene thioamide (12) based upon analytical and spectroscopic data, the second product (18\%) is formulated as 
2-(4-methoxyphenyl)-4-(4-thioxo-4H-chromen-3-yl)-1,2-thiaphosphetane-3,3-dicarbonitrile-2sulfide (14) (Scheme 3).

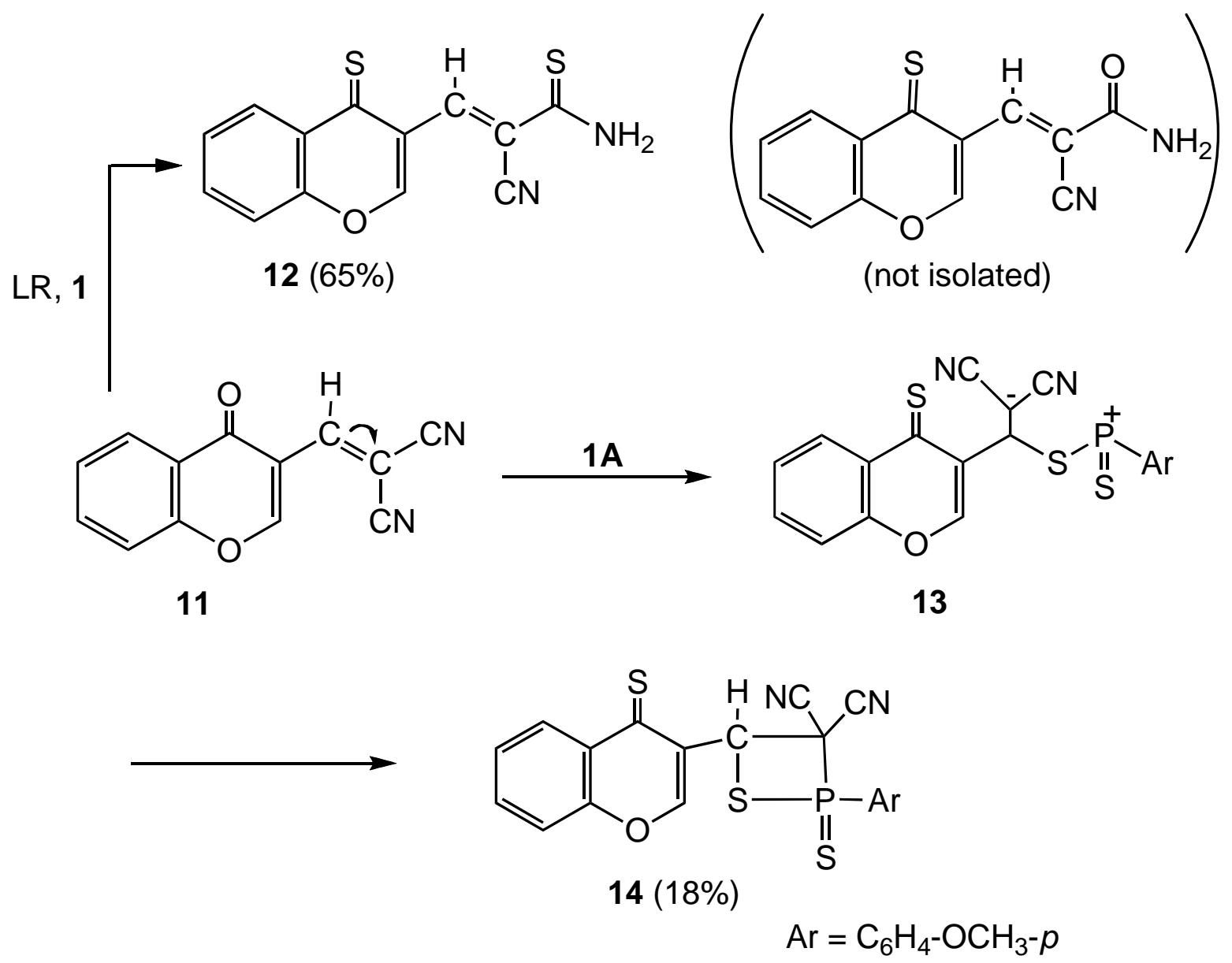

\section{Scheme 3}

The IR spectrum (in $\mathrm{KBr}, \mathrm{cm}^{-1}$ ) of compound 14-showed a strong absorption band at 2275 $\mathrm{cm}^{-1}$ corresponding to $(2 \mathrm{CN})$ groups and $1180 \mathrm{~cm}^{-1}(\mathrm{C}=\mathrm{S})$. Its ${ }^{1} \mathrm{H}$ NMR revealed presence of a signal at $6.39 \mathrm{ppm}\left(\mathrm{d},{ }^{3} J_{H P}=12 \mathrm{~Hz} 1 \mathrm{H}, \mathrm{P}-\mathrm{S}-\mathrm{CH}\right)$, a singlet at $3.75 \mathrm{ppm}$ corresponding to the $\mathrm{OCH}_{3}$ protons.

Formation of the thioamide derivative $\mathbf{1 2}$ can be attributed to a partial hydrolysis ${ }^{9,12}$ of $\mathbf{1 1}$ to yield the respective $\alpha$-cyano- $\beta$-substituted acrylamide intermediate which underwent ketone-tothioketone conversion under the thiating effect ${ }^{1-3}$ of LR to afford the thioamide chromene derivative 12.

The proposed mechanism for formation 14 involves initial nucleophilic attack by $1 \mathrm{~A}$ on $\mathbf{1 1}$ to give the transient intermediate 13. This process is followed by ring closure ${ }^{13}$ to give the thiaphosphetane chromene derivative 14.

Next, the reaction of ethyl (2E)-2-cyano-3-(4-oxo-4H-chromen-3-yl)acrylate 15 with Lawesson's reagent was carried out in boiling toluene, giving (5Z)-2-(4-methoxyphenyl)-5-[(4- 
thioxo-4H-chromen-3-yl)methylene]-1,3,2-thiazaphosphinane-4,6-dithione-2-sulfide (17) as a sole product (69\% yield) (Scheme 4).

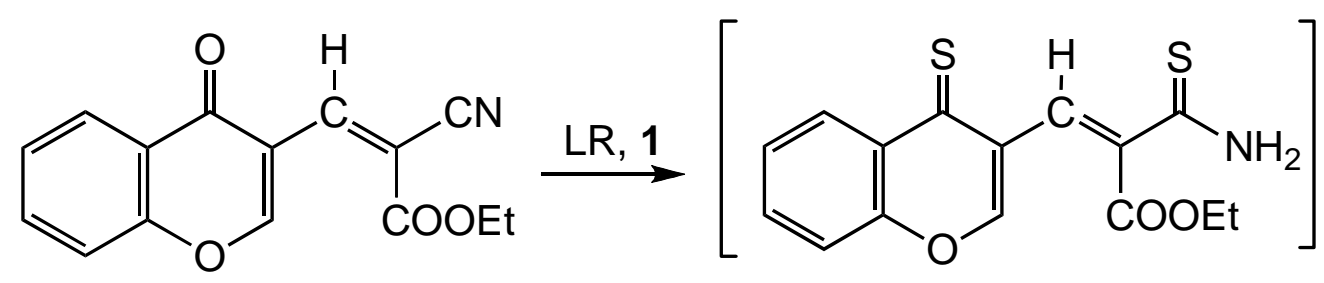

15

16

$1 \mathrm{~A}$

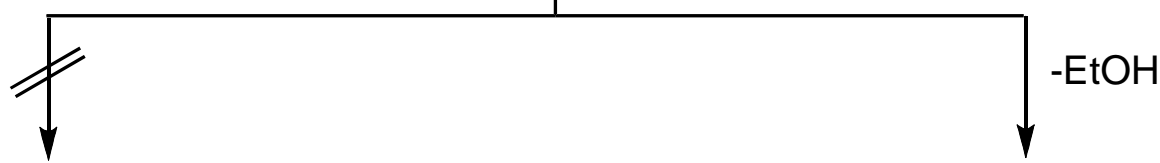<smiles>CCOC(=O)C1(C(N)=S)[C@H](c2coc3ccccc3c2=S)SP1(=S)[In]</smiles><smiles>[Al]P1(=S)NC(=S)C(=Cc2coc3ccccc3c2=S)C(=S)S1</smiles>

$17(69 \%)$

$$
\mathrm{Ar}=\mathrm{C}_{6} \mathrm{H}_{4}-\mathrm{OCH}_{3}-p
$$

\section{Scheme 4}

The structure of $\mathbf{1 7}$ was confirmed by analytical and spectral data which showed the disappearance of both the cyano and carboxylate groups. The ${ }^{1} \mathrm{H}$ NMR spectrum of $\mathbf{1 7}$ showed a singlet at 3.82 due to methoxy protons, also a singlet at 6.8 due to exocyclic ethylenic protons and a broad band at 10.94 due to the $\mathrm{NH}$ proton.

It is noteworthy that, in this reaction, the expected 1,2-thiaphosphetane derivative $\mathbf{1 8}$ was not formed. Instead, the thiazaphosphinane chromene 17 (69\% yield) was isolated in a pure form. It is believed to be formed via the thioamideacrylate intermediate $\mathbf{1 6}$ with concomitant elimination of an alcoholic moiety. Such cyclization reactions involving Lawesson's reagent leading to various phospha-heterocycles were previously discussed. ${ }^{14-16}$

Note: in all the above-mentioned reactions a colorless crystalline phosphorus-containing product was isolated (or detected by TLC) and proved to be trimer 19 by comparing its m.p. as well as IR and ${ }^{1} \mathrm{H}$ NMR spectra with those of an authentic specimen. ${ }^{17-19}$ Formation of $\mathbf{1 9}$ is frequently observed during thiation processes using LR. 


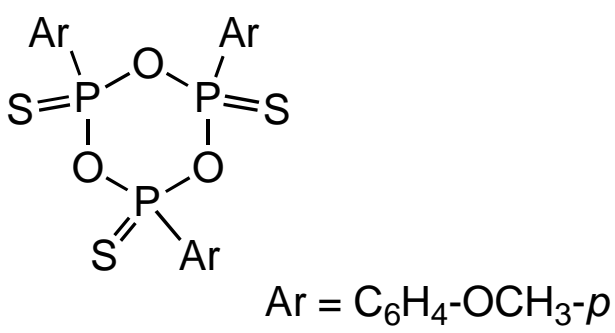

19

\section{Pharmacological evaluation}

The synthesized products $\mathbf{6}, \mathbf{8 b}, \mathbf{d}, \mathbf{1 0 b}, \mathbf{1 2}, \mathbf{1 4}, \mathbf{1 7}$ were screened against various types of fungi including $P$. brevicompactum, As. niger and As. fumigatus by adopting a food poisoning technique. Compounds $\mathbf{8 b}, \mathbf{d}$ and $\mathbf{1 2}$ are moderately active against $P$. brevicompactum and As. fumigatus at $400 \mathrm{mg} / \mathrm{mL}$ concentration level, while compounds $\mathbf{6}, \mathbf{1 0 b}, \mathbf{1 4}$, and 17 are more active against the same fungi at the same dose level.

Compounds 6 and 14 registered 100\% spore germination inhibition in As. niger at 500 $\mathrm{mg} / \mathrm{mL}$ whereas, compound $\mathbf{1 2}$ was found to have feeble activity.

The prepared products were also tested against one or the other type of bacteria including $B$. subitilis, B. cereus and E. coli. Compounds $\mathbf{8 b}, \mathbf{d}$ and $\mathbf{1 2}$ exhibited reasonable activities whereas, the phosphorylated derivatives $\mathbf{6 , 1 0 b}, \mathbf{1 4}$ and $\mathbf{1 7}$ showed the highest inhibitory effect against all the tested organisms, possibly attributable to the presence of the phosphorus moiety.

On the basis of our results, compounds 6, 10b, 14 and 17 would be good candidates, lead molecules to be modified in order to improve the anti-microbial activity.

\section{Conclusions}

In summary, the present investigations describe an efficient and simple approach to the synthesis of a variety of biologically active 4- and 6-membered P-S-heterocycles in satisfactory yields, with the use of easily available starting materials.

\section{Experimental Section}

General Procedures. All melting points are uncorrected. IR spectra were recorded on a PerkinElmer spectrophotometer model 297 using $\mathrm{KBr}$ disc. The ${ }^{1} \mathrm{H}$ and ${ }^{13} \mathrm{C}$ NMR spectra were recorded on a JNM-GX-400 Fa Joel spectrometer, using TMS as an internal reference. ${ }^{31} \mathrm{P}-\mathrm{NMR}$ spectra were taken with a Varian CFT-20 (vs. external $85 \% \mathrm{H}_{3} \mathrm{PO}_{4}$ ). The mass spectra were performed at $70 \mathrm{eV}$ on an MS-50 Kratos (A.E.I.) spectrometer provided with a data system. Elemental analyses were carried out at the Microanalysis Laboratory, Cairo University, Cairo, Egypt. The appropriate precautions in handing moisture-sensitive compounds were observed. 
Starting material $\mathbf{2}$ and LR, $\mathbf{1}$ were commercially available (From Aldrich Co.). Monoanils 7a,b were prepared as described by Fitton et al. ${ }^{20}$ The preparation of the new starting chromone derivatives 7c,d, 11 and $\mathbf{1 5}$ is described below (I, II, III).

Preparation of 3-[(Z)-[(4-aminophenyl)imino]methyl]-4H-chromene-4-one (7c) and 3-[(Z)[(2-aminophenyl)imino]methyl]-4H-chromene-4-one (7d). General procedure

A solution of equimolar quantities of 3-formylchromone (2) (10 g, $0.057 \mathrm{~mol})$ and 4aminophenylamine (in case of 7c), or 2-aminophenyl amine (in case of 7d) and one crystal of $p$ toluenesulfonic acid in dry benzene $(100 \mathrm{~mL})$ was heated under reflux (Dean-Stark water trap) for $30 \mathrm{~min}$. The solvent was evaporated off and the products were purified by crystallisation from ether to obtain $\mathbf{7 c}$ (m.p. $189-190{ }^{\circ} \mathrm{C}$ ) or $\mathbf{7 d}$ (m.p. $170-172{ }^{\circ} \mathrm{C}$ ) as red crystals (yield $\approx 85 \%$ ). Elemental analyses and spectral data for compounds $\mathbf{7 c}, \mathbf{7 d}$ were in agreement with the proposed structure. Elemental analyses for compound 7d: found C, 72.69; H, 4.50; N, 10.61\% $\mathrm{C}_{16} \mathrm{H}_{12} \mathrm{~N}_{2} \mathrm{O}_{2}$ (264.28) requires $\mathrm{C}, 72.72 ; \mathrm{H}, 4.58 ; \mathrm{N}, 10.60 \%$; IR $(\mathrm{v}): 1610(\mathrm{CH}=\mathrm{N}), 1530,1600(\mathrm{CH}=\mathrm{CH})$, $3300\left(\mathrm{NH}_{2}\right) ;{ }^{1} \mathrm{H}$ NMR $\left(\mathrm{CDCl}_{3}\right)$ gave signals at: $\delta 5.75$ (br.) Due to $\mathrm{NH}_{2}$, multiplet at 6.56-8.26 corresponding to the aromatic protons and a singlet $(1 \mathrm{H})$ at 7.68 due to the exocyclic ethylenic proton; $m / z$ (EI): $264\left[\mathrm{M}^{+}\right](26)$.

Preparation of [(4-oxo-4H-chromen-3-yl)methylene]malononitrile (11). A solution of 2 (10 g, $0.057 \mathrm{~mol})$, malononitrile $(0.1 \mathrm{~mol})$, ammonium acetate $(20 \mathrm{~g})$ and glacial acetic acid $(50 \mathrm{~mL})$ in $200 \mathrm{~mL}$ of toluene was refluxed for $10 \mathrm{~h}$ with the aid of a Dean-Stark trap until the amount of water collected in the trap remained constant. The solid formed was collected then recrystallized from cyclohexane to give $\mathbf{1 1}$ as yellow crystals (yield 79\%), m.p 186-187 ${ }^{\circ} \mathrm{C}$. Elemental analyses found: $\mathrm{C}, 70.31 ; \mathrm{H}, 2.68 ; \mathrm{N}, 12.45 \% \mathrm{C}_{13} \mathrm{H}_{6} \mathrm{~N}_{2} \mathrm{O}_{2}(222.207)$ requires: $\mathrm{C}, 70.27 ; \mathrm{H}, 2.73 ; \mathrm{N}$, $12.61 \% ; v_{\max }(\mathrm{KBr}) / \mathrm{cm}^{-1}: 1650(\mathrm{C}=\mathrm{O}), 1600(\mathrm{C}=\mathrm{C}), 2210(\mathrm{C} \equiv \mathrm{N}) ; \delta_{H}(\mathrm{ppm})$ showed a multiplet at 7.53-8.28 due to the aromatic protons wherein gave a singlet $(1 \mathrm{H})$ at 7.20 due to the exocyclic ethylenic proton; $m / z(E I): 222\left[\mathrm{M}^{+}\right](28)$.

Preparation of ethyl (2E)-2-cyano-3-(4-oxo-4H-chromen-3-yl)acrylate (15). A solution of 2 $(10 \mathrm{~g}, 0.057 \mathrm{~mol})$, ethyl cyanoacetate $(0.1 \mathrm{~mol})$, ammonium acetate $(20 \mathrm{~g})$ and glacial acetic acid $(50 \mathrm{~mL})$ in $200 \mathrm{~mL}$ toluene was refluxed for $18 \mathrm{~h}$ with the aid of a Dean-Stark trap. The solid formed was collected then recrystallized from ethanol to give compound $\mathbf{1 5}$ as pale yellow crystals (yield $85 \%$ ), m.p $240-241{ }^{\circ} \mathrm{C}$. Elemental analyses found: C, 66.81; H, 4.35; N, 5.41\%

$\mathrm{C}_{15} \mathrm{H}_{11} \mathrm{NO}_{4}(269.26)$ requires: $\mathrm{C}, 66.91 ; \mathrm{H}, 4.12 ; \mathrm{N}, 5.20 \% ; v_{\max }(\mathrm{KBr}) / \mathrm{cm}^{-1}: 1730(\mathrm{C}=\mathrm{O}$, acetate), $1560(\mathrm{C}=\mathrm{C}$, aromatic $), 2200(\mathrm{C} \equiv \mathrm{N}) ; \delta_{H}(\mathrm{ppm})$ showed a multiplet at 7.61-8.32 due to the aromatic protons and a singlet $(1 \mathrm{H})$ at 7.64 due to the exocyclic ethylenic proton, $1.24[\mathrm{t}, 3 \mathrm{H}$, $\left(-\mathrm{C}-\mathrm{CH}_{3}\right)$ ], 4.2 [q, 2H, $\left.\left(\mathrm{O}-\mathrm{CH}_{2}\right)\right] ; \mathrm{m} / \mathrm{z}(\mathrm{EI}): 269\left[\mathrm{M}^{+}\right](22)$.

Reaction of 2 with Lawesson's reagent 1. Preparation of compounds 3 and 6. General procedure

To a stirred solution $(2 \mathrm{~g}, 11.4 \mathrm{mmol})$ of $\mathbf{2}$ in dry toluene $(25 \mathrm{~mL})$, Lawesson's reagent $\mathbf{1}(1.4 \mathrm{~g}$, $5.9 \mathrm{mmol}$ ) was added. The reaction mixture was refluxed with stirring for $4 \mathrm{~h}$. The reaction 
mixture was evaporated under reduced pressure. The resulting residue was chromatographed on silica gel using increasing amounts of -hexane/AcOEt as eluents to give compounds $\mathbf{3}$ and $\mathbf{6}$.

4-Thioxo-4H-chromene-3-carbothialdehyde (3) was obtained (9:1 v/v) as pale yellow crystals (20\% yield), m.p. 130-132 ${ }^{\circ} \mathrm{C}$ (from cyclohexane). Found: C, 58.43; H, 3.0; S, 31.35\%; $\mathrm{C}_{10} \mathrm{H}_{6} \mathrm{OS}_{2}(206.29)$ requires; $\mathrm{C}, 58.22 ; \mathrm{H}, 2.93 ; \mathrm{S}, 31.09 \% ; v_{\max }(\mathrm{KBr}) / \mathrm{cm}^{-1}: 1100(\mathrm{C}=\mathrm{S}$, thioxo) $1190\left(\mathrm{C}=\mathrm{S}\right.$, ald.), $1600\left(\mathrm{C}=\mathrm{C}\right.$, aromatic ring); $\delta_{H}(\mathrm{ppm}): 7.3,8.74\left(2 \mathrm{~d}, J_{H H}=7.5 \mathrm{~Hz}, 2 \mathrm{H}, \mathrm{CH}-\mathrm{ph}\right)$, 7.28, 7.34 (2t, 2H, CH-ph ), $9.67(\mathrm{~s}, 1 \mathrm{H}, \mathrm{CH}-\mathrm{O}), 10.6(\mathrm{~s}, 1 \mathrm{H}, \mathrm{HC}=\mathrm{S}) ; \delta_{C}(\mathrm{ppm}) 196.21(\mathrm{C}=\mathrm{S}$ ring), $186.45(\mathrm{H}-\mathrm{C}=\mathrm{S}), 119.88,125.66,129.53,133.25,134.55,152.33,162.40$ (aromatic carbon atoms); $m / z,(\mathrm{El}): 206\left[\mathrm{M}^{+}\right](22)$.

2-(4-Methoxyphenyl)-5H,10aH-[1,3,2]oxathiaphosphinino[4,5-b]chromene-5-thione-2sulfide (6) was obtained (8:2 v/v) as yellow crystals (60\% yield), m.p. $180-182{ }^{\circ} \mathrm{C}$ (from ether). Found: C, 52.34; H, 3.11; P, 8.0; S, 24.73\% $\mathrm{C}_{17} \mathrm{H}_{13} \mathrm{O}_{3} \mathrm{PS}_{3}(392.47)$ requires $\mathrm{C}, 52.03 ; \mathrm{H}, 3.34 ; \mathrm{P}$, 7.89; S, 24.51\%; $v_{\max }(\mathrm{KBr}) / \mathrm{cm}^{-1} 1220(\mathrm{C}=\mathrm{S}) 1580,1600\left(\mathrm{C}=\mathrm{C}\right.$, aromatic) $650(\mathrm{P}=\mathrm{S}) ; \delta_{H}(\mathrm{ppm})$ : $3.85\left(\mathrm{~s}, 3 \mathrm{H}, \mathrm{OCH}_{3}\right), 7.4\left(\mathrm{~d},{ }^{3} J_{H P}=11.3,1 \mathrm{H}, \mathrm{P}-\mathrm{S}-\mathrm{CH}\right), 6.95$ and $7.56\left(2 \mathrm{dd}, 4 \mathrm{H}, J_{H H}=9 \mathrm{~Hz},{ }^{3} J_{H P}\right.$ $=11 \mathrm{~Hz}, H$-Ar $), 7.95,8.7\left(2 \mathrm{~d}, 2 \mathrm{H}, J_{H H}=7 \mathrm{~Hz}, \mathrm{H}-\mathrm{Ph}\right), 6.8,7.1(2 \mathrm{t}, 2 \mathrm{H},=\mathrm{CH}-\mathrm{CH}=), 8.8\left(\mathrm{~d},{ }^{3} J_{H P}=\right.$ $10.5,1 \mathrm{H}, \mathrm{P}-\mathrm{O}-\mathrm{CH}=\mathrm{C}) ; \delta_{C}(\mathrm{ppm}): \delta 55.30\left(\mathrm{OCH}_{3}\right), 84.71\left(\mathrm{~d},{ }^{2} J_{C P}=40.1, \mathrm{O}-\mathrm{CH}-\mathrm{S}\right), 112.05(\mathrm{~S}=\mathrm{C}-$ $C=\mathrm{C}), 112.88,119.06,124.22,125.66,129.53,132.51,134.51,134.55,150.66,151.11,152.33$, $158.80\left(C=C\right.$, aromatic), $150.57\left(\mathrm{~d},{ }^{2} J_{C P}=38.9, \mathrm{C}=C-\mathrm{O}-\mathrm{P}=\mathrm{S}\right), 216.77(C=\mathrm{S}) ; \delta_{P}\left(\mathrm{CDCl}_{3}\right): 93.39$ ppm; $m / z(\mathrm{El}): 392\left[\mathrm{M}^{+}\right](26)$.

Reaction of 3-(aryliminomethyl)chromones 7a-d with 1. Preparation of compounds 8a-d and 10a-d. To a stirred solution $(11.4 \mathrm{mmol})$ of 3-(aryliminomethyl) chromones 7a-d in dry toluene $(25 \mathrm{~mL})$, Lawesson's reagent 1 (1.4 g, $5.9 \mathrm{mmol})$ was added. The reaction mixture was refluxed for 6-8 $\mathrm{h}$ until the reaction was finished (TLC). Working up the product mixture as described in the general procedure and column chromatography furnished compounds 8a-d and 10a-d.

3-[(E)-[(4-Chlorophenyl)imino]methyl]-4H-chromene-4-thione (8a) was obtained (8:2 v/v ) as pale yellow crystals ( $15 \%$ yield), m.p $136-138{ }^{\circ} \mathrm{C}$ (from cyclohexane). Found: C, 64.50; H, 3.12; $\mathrm{Cl}, 11.72 ; \mathrm{N}, 4.60 ; \mathrm{S}, 10.62 \% \mathrm{C}_{16} \mathrm{H}_{10} \mathrm{ClNOS}$ (299.78) requires $\mathrm{C}, 64.11 ; \mathrm{H}, 3.36 ; \mathrm{Cl}, 11.83 ; \mathrm{N}$, 4.67; S, 10.70\%; $v_{\max }(\mathrm{KBr}) / \mathrm{cm}^{-1}: 1610(\mathrm{CH}=\mathrm{N}),(\mathrm{CH}=\mathrm{CH}) 1570,1600 ; \delta_{H}(\mathrm{ppm}): \delta 7.28(\mathrm{~S}, \mathrm{H}$, $\mathrm{CH}=\mathrm{N}), 6.48,7.21\left(2 \mathrm{~d}, 4 \mathrm{H}, J_{H H}=8.8 \mathrm{~Hz}, \mathrm{H}-\mathrm{ph}-\mathrm{Cl}\right), 7.51,8.66\left(2 \mathrm{~d}, 2 \mathrm{H}, J_{H H}=7.3 \mathrm{~Hz}, \mathrm{CH}-\mathrm{ph}\right)$, 7.24, $7.48(2 \mathrm{t}, 2 \mathrm{H},=\mathrm{CH}-\mathrm{CH}=), 8.87(\mathrm{~s}, 1 \mathrm{H},=\mathrm{CH}-\mathrm{O}) ; \delta_{C}(\mathrm{ppm}): 119.55,121.80,122.16,124.04$, $125.22,132.1,128.38,135.33,142.35,148.05,151.18,151.93,166.20$, (aromatic carbon atoms), 199.69 ( $C=\mathrm{S}$ ring); $\mathrm{m} / \mathrm{z}(\mathrm{EI}): 299\left[\mathrm{M}^{+}\right](16)$.

3-[(E)[4-Methoxyphenyl]imino\}methyl]-4H-chromene-4-thione (8b) was obtained (8:2 v/v) as pale yellow crystals (18\%, yield), m.p. $134-136{ }^{\circ} \mathrm{C}$ (from $n$-hexane). Found: $\mathrm{C}, 69.43$; H, 4.12; $\mathrm{N}, 5.00 ; \mathrm{S}, 10.92 \% \mathrm{C}_{17} \mathrm{H}_{13} \mathrm{NO}_{2} \mathrm{~S}(295.37)$ requires $\mathrm{C}, 69.13 ; \mathrm{H}, 4.44 ; \mathrm{N}, 4.74 ; \mathrm{S}, 10.86 \%$; $v_{\max }(\mathrm{KBr}) / \mathrm{cm}^{-1}(\mathrm{CH}=\mathrm{N}), 1615,(\mathrm{CH}=\mathrm{CH}) 1540,1600 ; \delta_{H}(\mathrm{ppm}): 3.77\left(\mathrm{~s}, 3 \mathrm{H},-\mathrm{OCH}_{3}\right), 7.28(\mathrm{~s}$, $1 \mathrm{H}, \mathrm{CH}=\mathrm{N}), 6.53,7.19\left(2 \mathrm{~d}, 4 \mathrm{H}, J_{H H}=8.6 \mathrm{~Hz}, \mathrm{H}-\mathrm{Ph}-\mathrm{OCH}_{3}\right), 7.5,8.67\left(2 \mathrm{~d}, 2 \mathrm{H}, J_{H H}=7.5 \mathrm{~Hz}\right.$, $\mathrm{CH}$-ph), $7.23,7.31(2 \mathrm{t}, 2 \mathrm{H},=\mathrm{CH}=\mathrm{CH}=), 8.87(\mathrm{~s}, 1 \mathrm{H},=\mathrm{CH}-\mathrm{O}) ; \delta_{C}(\mathrm{ppm}): 114.49,119.23,122.75$, 
125.23, 128.99, 132.33, 135.45, 142.22, 148.05, 151.42, 155.82, 156.12, 166.20 (aromatic carbon atoms), 199.69 (C=S, ring) ; m/z (EI): $295\left[\mathrm{M}^{+}\right]$(22).

3-[(E)-[(4-Aminophenyl)imino]methyl]-4H-chromene-4-thione (8c) was obtained (8:2 v/v) as pale yellow crystals ( $20 \%$ yield), m.p. $138-140{ }^{\circ} \mathrm{C}$ (from cyclohexane). Found: $\mathrm{C}, 68.64 ; \mathrm{H}, 4.58$; $\mathrm{N}, 10.02 ; \mathrm{S}, 11.29 \% \mathrm{C}_{16} \mathrm{H}_{12} \mathrm{~N}_{2} \mathrm{OS}(280.35)$ requires $\mathrm{C}, 68.55 \mathrm{H}, 4.31 ; \mathrm{N}, 9.99 ; \mathrm{S}, 11.44 \%$; $\nu_{\max }(\mathrm{KBr}) / \mathrm{cm}^{-1}: 1620(\mathrm{CH}=\mathrm{N}), 1570,1600(\mathrm{CH}=\mathrm{CH}), 3350,3400\left(\mathrm{NH}_{2}\right) ; \delta_{H}(\mathrm{ppm}): 5.85(\mathrm{~s}, 2 \mathrm{H}$, $\left.\mathrm{NH}_{2}\right), 7.43(\mathrm{~s}, \mathrm{H}, \mathrm{CH}=\mathrm{N}), 6.42,7.44\left(2 \mathrm{~d}, 4 \mathrm{H}, J_{\mathrm{HH}}=8.6 \mathrm{~Hz}, H-\mathrm{Ph}-\mathrm{NH}_{2}\right), 7.55,8.69\left(2 \mathrm{~d}, 2 \mathrm{H}, J_{\mathrm{HH}=}\right.$ $7.5 \mathrm{~Hz}, \mathrm{CH}-\mathrm{ph}), 7.25,7.47(2 \mathrm{t}, 2 \mathrm{H},=\mathrm{CH}-\mathrm{CH}=), 8.75(\mathrm{~s}, 1 \mathrm{H}, \mathrm{CH}-\mathrm{O}) ; \delta_{C}(\mathrm{ppm}): 108.55,110.55$, $119.07,122.35,125.27,128.68,135.33,132.20,134.50,142.11,148.05,151.18,166.20$ (aromatic carbon atoms), $199.42\left(\mathrm{C}=\mathrm{S}\right.$, ring); $\mathrm{m} / \mathrm{z}(\mathrm{EI}): 280\left[\mathrm{M}^{+}\right](19)$.

3-[(E)-[(2-Aminophenyl)imino]methyl]-4H-chromene-4-thione (8d) was obtained $(8: 2 \mathrm{v} / \mathrm{v})$ as yellow crystals (18\% yield), m.p $142-144{ }^{\circ} \mathrm{C}$ (from cyclohexane). Found: $\mathrm{C}, 68.31 ; \mathrm{H}, 4.59 ; \mathrm{N}$, 9.82; S, 11.64\% $\mathrm{C}_{16} \mathrm{H}_{12} \mathrm{~N}_{2} \mathrm{OS}$ (280.35) requires $\mathrm{C}, 68.55 ; \mathrm{H}, 4.31 ; \mathrm{N}, 9.99 ; \mathrm{S}, 11.44 \%$; $v_{\max }(\mathrm{KBr}) / \mathrm{cm}^{-1}: 1615(\mathrm{CH}=\mathrm{N}), 1540,1600(\mathrm{CH}=\mathrm{CH}), 3320,3400\left(\mathrm{NH}_{2}\right) ; \delta_{H}(\mathrm{ppm}): 3.71(\mathrm{~s}, 2 \mathrm{H}$, $\left.\mathrm{NH}_{2}\right), 7.28(\mathrm{~s}, \mathrm{H}, \mathrm{CH}=\mathrm{N}), 6.26,6.31\left(2 \mathrm{t}, 2 \mathrm{H}, \mathrm{H}-\mathrm{ph}-\mathrm{NH}_{2}\right), 6.45,6.92\left(2 \mathrm{~d}, 2 \mathrm{H}, J_{\mathrm{HH}}=7.2 \mathrm{~Hz}, H\right.$-ph$\left.\mathrm{NH}_{2}\right), 7.23,7.41(2 \mathrm{t}, 2 \mathrm{H},=\mathrm{CH}-\mathrm{CH}=), 7.47,8.21\left(2 \mathrm{~d}, 2 \mathrm{H}, J_{\mathrm{HH}}=7.64 \mathrm{~Hz}, \mathrm{CH}-\mathrm{ph}\right), 8.87(\mathrm{~s}, 1 \mathrm{H}$, $=\mathrm{CH}-\mathrm{O}) ; \delta_{\mathrm{C}}(\mathrm{ppm}): 119.07,120.64,122.20,123.06,125.27,131.30,135.34,140.79$, $142.15,149.49,151.20,151.85,166.20$ (aromatic carbon atoms), 198.65 (-C=S ring); $\mathrm{m} / \mathrm{z}(\mathrm{EI})$ : $280\left[\mathrm{M}^{+}\right](16)$.

3-[3-(4-Chlorophenyl)-2-(4-methoxyphenyl)-2-sulfido-1,3,2-thiazaphosphetidin-4-yl]-4Hchromene-4-thione (10a) was obtained (6:4 v/v) as yellow crystals (45\% yield) m.p $155-157{ }^{\circ} \mathrm{C}$ (from ether). Found: C, 55.24; $\mathrm{H}, 3.63 ; \mathrm{Cl}, 6.99 ; \mathrm{N}, 2.58 ; \mathrm{P}, 6.43 ; \mathrm{S}, 19.24 \%$ $\mathrm{C}_{23} \mathrm{H}_{17} \mathrm{ClNO}_{2} \mathrm{PS}_{3}(502.03)$ requires $\mathrm{C}, 55.03 ; \mathrm{H}, 3.41 ; \mathrm{Cl}, 7.06 ; \mathrm{N}, 2.79 ; \mathrm{P}, 6.17 ; \mathrm{S}, 19.16 \%$; $v_{\max }(\mathrm{KBr}) / \mathrm{cm}^{-1}: 1190(\mathrm{C}=\mathrm{S}), 1530-1600(\mathrm{CH}=\mathrm{CH}), 653(\mathrm{P}=\mathrm{S}): \delta_{H}(\mathrm{ppm}): 3.74\left(\mathrm{~s}, 3 \mathrm{H}, \mathrm{OCH}_{3}\right)$, $5.89\left(\mathrm{~d}, 1 \mathrm{H},{ }^{3} J_{H P}=12 \mathrm{~Hz}, \mathrm{~S}-\mathrm{CH}-\mathrm{N}\right) ; 6.99-8.75$ (m, 13H, aromatics); $\delta_{C}(\mathrm{ppm}): 56.05\left(-\mathrm{O}-\mathrm{CH}_{3}\right)$, $67.19\left(\mathrm{~d},{ }^{2} J_{C P}=43, \mathrm{P}-\mathrm{S}-\mathrm{C}-\mathrm{N}\right), 113.53,118.38,119.66,120.53,123.87,130.50,131.89,138.55$, 139.22, 134.64, 136.02, 140.99, 141.52, 149.85, 153.40, 160.95 (aromatic carbon atoms), 205.68 $\left(C=\mathrm{S}\right.$, ring); $\delta_{P}\left(\mathrm{CDCl}_{3}\right): 48.43 \mathrm{ppm} ; \mathrm{m} / \mathrm{z}(\mathrm{EI}): 502\left[\mathrm{M}^{+}\right](26)$.

3-[3-(4-Methoxyphenyl)-2-(4-methoxyphenyl)-2-sulfido-1,3,2-thiazaphosphetidin-4-yl]-4Hchromene-4-thione (10b) was obtained $(1: 1 \mathrm{v} / \mathrm{v})$ as yellow crystals $\left(50 \%\right.$ yield) m.p $158-160{ }^{\circ} \mathrm{C}$ (from acetone / ether). Found: C, 57.66; H, 4.34; N, 3.01; P,6.49; S, 19.55\% $\mathrm{C}_{24} \mathrm{H}_{20} \mathrm{NO}_{3} \mathrm{PS}_{3}(497.61)$ requires $\mathrm{C}, 57.93 ; \mathrm{H}, 4.05 ; \mathrm{N}, 2.82 ; \mathrm{P}, 6.23 ; \mathrm{S}, 19.33 \% ; v_{\max }(\mathrm{KBr}) / \mathrm{cm}^{-1}$ : 1570, $1600(\mathrm{CH}=\mathrm{CH}), 1200(\mathrm{C}=\mathrm{S}), 645(\mathrm{P}=\mathrm{S}) ; \delta_{H}(\mathrm{ppm}) 3.50\left(\mathrm{~s}, 3 \mathrm{H},\left(\mathrm{N}-\mathrm{Ph}-\mathrm{OCH}_{3}\right), 3.82(\mathrm{~s}, 3 \mathrm{H}\right.$, $\left(\mathrm{P}-\mathrm{Ph}-\mathrm{OCH}_{3}\right), 5.34\left(\mathrm{~d}, 1 \mathrm{H},{ }^{3} J_{H P}=12 \mathrm{~Hz}, \mathrm{~S}-\mathrm{CH}-\mathrm{N}\right), 6.45-8.32\left(\mathrm{~m}, 13 \mathrm{H}\right.$, aromatics); $\delta_{C}(\mathrm{ppm})$ : $54.59\left(\mathrm{P}-\mathrm{Ph}-\mathrm{OCH}_{3}\right), 55.21\left(\mathrm{~N}-\mathrm{Ph}-\mathrm{OCH}_{3}\right), 67.57\left[\mathrm{~d},{ }^{2} J_{C P}=42,(\mathrm{PS}-\mathrm{CH}-\mathrm{N})\right], 113.56,115.35$, $119.45,122.56,124.92,129.84,130.12,132.55,134.64,135.92,139.99,141.35,144.81,149.85$, 153.40, 155.55, 158.34 (aromatic carbon atoms), $205.67\left(C=\mathrm{S}\right.$, ring); $\delta_{P}\left(\mathrm{CDCl}_{3}\right)$ at $48.92 \mathrm{ppm}$; $\mathrm{m} / \mathrm{z}(\mathrm{EI}): 497\left[\mathrm{M}^{+}\right](18)$.

3-[3-(4-Aminophenyl)-2-(4-methoxyphenyl)-2-sulfido-1,3,2-thiazaphosphetidin-4-yl]-4Hchromene-4-thione (10c) was obtained $(1: 1 \mathrm{v} / \mathrm{v})$ as yellow crystals $(59 \%$ yield $) \mathrm{m} . \mathrm{p} 165-166^{\circ} \mathrm{C}$ 
(from acetone / ether). Found: C, 56.99; H, 3.79; N, 5.53; P, 6.26; S, $19.81 \% \mathrm{C}_{23} \mathrm{H}_{19} \mathrm{~N}_{2} \mathrm{O}_{2} \mathrm{PS}_{3}$ (482.58) requires $\mathrm{C}, 57.24 ; \mathrm{H}, 3.97 ; \mathrm{N}, 5.8 ; \mathrm{P}, 6.42 ; \mathrm{S}, 19.93 \% ; \mathrm{v}_{\max }(\mathrm{KBr}) / \mathrm{cm}^{-1}: 1560,1600$ $\left(\mathrm{CH}=\mathrm{CH}\right.$, aromatic), $3200\left(\mathrm{NH}_{2}\right), 1190(\mathrm{C}=\mathrm{S}), 650(\mathrm{P}=\mathrm{S}) ; \delta_{H}(\mathrm{ppm}): 3.82\left(\mathrm{~s}, 3 \mathrm{H},-\mathrm{OCH}_{3}\right), 5.99(\mathrm{~s}$, $\left.2 \mathrm{H},-\mathrm{NH}_{2}\right), 6.03\left(\mathrm{~d}, 1 \mathrm{H},(\mathrm{S}-\mathrm{CH}-\mathrm{N}),{ }^{3} J_{H P}=12 \mathrm{~Hz}\right), 6.5-8.32\left(\mathrm{~m}, 13 \mathrm{H}\right.$, aromatics); $\delta_{C}(\mathrm{ppm}): 54.83$ $\left(\mathrm{Ph}-\mathrm{OCH}_{3}\right), 67.19$ (d, ${ }^{2} J_{C P}=40$, PS-CH-N), 115.89, 117.68, 118.33,120.23, 124.82,122.88, $128.53,129.64,130.15,132.11,134.22,140.03,141.17,149.58,153.40,158.34$ (aromatic carbon atoms), $205.83\left(C=\mathrm{S}\right.$, ring); $\delta_{P}\left(\mathrm{CDCl}_{3}\right) 47.29 \mathrm{ppm} ; \mathrm{m} / \mathrm{z}(\mathrm{EI}): 482\left[\mathrm{M}^{+}\right](16)$.

3-[3-(2-Aminophenyl)-2-(4-methoxyphenyl)-2-sulfido-1,3,2-thiazaphosphetidin-4-yl]-4H-

chromene-4-thione (10d) was obtained (6:4 v/v)as yellow crystals $\left(50 \%\right.$ yield) m.p $159-160{ }^{\circ} \mathrm{C}$ (from acetone). Found: C, 57.59; H, 4.05; N, 5.48; P, 6.58; S, 19.80\% $\mathrm{C}_{23} \mathrm{H}_{19} \mathrm{~N}_{2} \mathrm{O}_{2} \mathrm{PS}_{3}(482.58)$ requires C, 57.24; H, 3.97; N, 5.8; P, 6.42; S, 19.93\%; IR $v_{\max }(\mathrm{KBr}) / \mathrm{cm}^{-1}: 1560,1600(\mathrm{CH}=\mathrm{CH}$, aromatic), $3300\left(\mathrm{NH}_{2}\right), 1180(\mathrm{C}=\mathrm{S}), 640(\mathrm{P}=\mathrm{S}): \delta_{H}(\mathrm{ppm}): 3.82\left(\mathrm{~s}, 3 \mathrm{H},-\mathrm{OCH}_{3}\right), 4.16(\mathrm{~s}, 2 \mathrm{H},-$ $\left.\mathrm{NH}_{2}\right), 6.51\left(\mathrm{~d}, 1 \mathrm{H},{ }^{3} J_{H P}=12 \mathrm{~Hz}, \mathrm{~S}-\mathrm{CH}-\mathrm{N}\right), 6.81-8.32\left(\mathrm{~m}, 13 \mathrm{H}\right.$, aromatics); $\delta_{C}(\mathrm{ppm}): 54.69(\mathrm{Ph}-$ $\left.\mathrm{OCH}_{3}\right), 67.34\left(\mathrm{~d},{ }^{2} J_{C P}=43\right.$, PS-CH-N), 105.09, 115.78, 116.99, 118.20, 120.23, 122.65, 124.12, $125.91,129.85,134.55,136.11,139.11,144.08,147.16,149.59,158.34$ (aromatic carbon atoms), $204.89\left(C=\mathrm{S}\right.$, ring); $\delta_{P}\left(\mathrm{CDCl}_{3}\right): 48.11 \mathrm{ppm} ; \mathrm{m} / \mathrm{z}(\mathrm{EI}): 482\left[\mathrm{M}^{+}\right](22)$.

\section{Reaction of 11 with Lawesson's reagent 1. Preparation of compounds 12 and 14}

To a stirred solution $(11.4 \mathrm{mmol})$ of [(4-oxo-4H-chromen-3-yl) methylene] malononitrile 10 in dry toluene $(25 \mathrm{~mL})$, Lawesson's reagent 1 (1.4 g, $5.9 \mathrm{mmol})$ was added. The reaction mixture was refluxed for $8 \mathrm{~h}$ until the reaction was completed (TLC). Working up the product mixture as described in the general procedure and column chromatography furnished compounds 12 and 14. (2E)-2-Cyano-3-(4-thioxo-4H-chromen-3-yl)prop-2-enethioamide (12) was obtained (8:2 v/v) as colorless crystals (65\% yield) m.p $152-154{ }^{\circ} \mathrm{C}$ (from cyclohexane). Found: C, 57.11, H, 2.87, $\mathrm{N}, 10.59, \mathrm{~S}, 23.22 \% \mathrm{C}_{13} \mathrm{H}_{8} \mathrm{~N}_{2} \mathrm{OS}_{2}(272.36)$ requires $\mathrm{C}, 57.33, \mathrm{H}, 2.96, \mathrm{~N}, 10.29, \mathrm{~S}, 23.55 \%$; $v_{\max }(\mathrm{KBr}) / \mathrm{cm}^{-1}: 3420,3390\left(\mathrm{NH}_{2}\right), 2200(\mathrm{C} \equiv \mathrm{N}), 1640(\mathrm{C}=\mathrm{C}$, ethylenic $), 1230(\mathrm{C}=\mathrm{S}), 1190$ $\left(\mathrm{C}=\mathrm{S}\right.$, ring), $1580\left(\mathrm{C}=\mathrm{C}\right.$, aromatic); $\delta_{H}(\mathrm{ppm}): 6.76(\mathrm{~s}, 1 \mathrm{H}, \mathrm{S}=\mathrm{C}-\mathrm{CH}=\mathrm{CH}), 7.46,8.85(2 \mathrm{~d}, 2 \mathrm{H}$, $\left.J_{H H}=7.5 \mathrm{~Hz}, \mathrm{CH}-\mathrm{ph}\right), 7.36,7.39(2 \mathrm{t}, 2 \mathrm{H}, \mathrm{CH}-\mathrm{ph}), 9.20(\mathrm{~s}, 1 \mathrm{H}, \mathrm{CH}-\mathrm{O}),\left(\mathrm{s}, 2 \mathrm{H}, \mathrm{NH}_{2}\right.$, exchangeable with $\left.\mathrm{D}_{2} \mathrm{O}\right) ; \delta_{C}(\mathrm{ppm}): 85.33,112.73,118.55,122.96,124.75,130.86,140.34$, 153.77, 161.86 (aromatic and exocyclic carbon atoms), $112.89(\mathrm{C} \equiv \mathrm{N}), 196.45(\mathrm{C}=\mathrm{S}$, thioamide), 201.26 (-C=S, ring); $m / z$ (EI): $272\left[\mathrm{M}^{+}\right](35)$.

\section{2-(4-Methoxyphenyl)-4-(4-thioxo-4H-chromen-3-yl)-1,2-thiaphosphetane-3,3-}

dicarbonitrile-2-sulfide (14) was obtained (1:1 v/v) as pale yellow crystals (18\% yield) m.p 165-163 ${ }^{\circ} \mathrm{C}$ (from acetone / ether). Found: C, 54.68; H, 3.04; N, 6.56; P, 7.33; S, 21.69\% $\mathrm{C}_{20} \mathrm{H}_{13} \mathrm{~N}_{2} \mathrm{O}_{2} \mathrm{PS}_{3}(440.52)$ requires $\mathrm{C}, 54.53 ; \mathrm{H}, 2.97 ; \mathrm{N}, 6.36 ; \mathrm{P}, 7.03 ; \mathrm{S}, 21.84 \% ; v_{\max }(\mathrm{KBr}) / \mathrm{cm}^{1}$ : $2275(\mathrm{C} \equiv \mathrm{N}), 1580(\mathrm{C}=\mathrm{C}$, aromatic $), 1180(\mathrm{C}=\mathrm{S}), 680(\mathrm{P}=\mathrm{S}) ; \delta_{H}(\mathrm{ppm}): 3.75\left(\mathrm{~s}, 3 \mathrm{H}, \mathrm{OCH}_{3}\right), 6.39$ $\left(\mathrm{d}, 1 \mathrm{H},{ }^{3} J_{H P}=12 \mathrm{~Hz}(\mathrm{~S}-\mathrm{CH}-\mathrm{C}), 6.8,7.87\left(2 \mathrm{dd}, 4 \mathrm{H}, J_{H H}=8.5 \mathrm{~Hz},{ }^{3} J_{H P}=10.5 \mathrm{~Hz}, H-\mathrm{Ar}\right), 6.85,7.9\right.$ $\left(2 \mathrm{~d}, 2 \mathrm{H}, J_{H H}=8.2 \mathrm{~Hz}, \mathrm{H}-\mathrm{Ph}\right), 7.45,7.55(2 \mathrm{t}, 2 \mathrm{H},=\mathrm{CH}-\mathrm{CH}=), 8.92(\mathrm{~s}, 1 \mathrm{H}, \mathrm{CH}-\mathrm{O}) ; \delta_{C}(\mathrm{ppm})$ : $40.59\left(\mathrm{~d}, J_{C P}=198 \mathrm{~Hz}, C-\mathrm{C} \equiv \mathrm{N}\right), 42.26\left(\mathrm{~d},{ }^{2} J_{C P}=32 \mathrm{~Hz}, \mathrm{P}-\mathrm{S}-\mathrm{CH}\right), 54.69\left(\mathrm{OCH}_{3}\right), 109.50,111.60$, $\left(2 \mathrm{~d},{ }^{2} \mathrm{~J}_{C P}=30 \mathrm{~Hz}, 2 \times \mathrm{C} \equiv \mathrm{N}\right), 115.54,116.70,119.20,120.89,124.30,130.89,131.51,131.85$, 
132.60, 134.20, 149.54, 153.70, 161.64 (aromatic carbon atoms), $209.11(\mathrm{C}=\mathrm{S}) ; \delta_{P}\left(\mathrm{CDCl}_{3}\right)$ : $50.12 \mathrm{ppm} ; \mathrm{m} / \mathrm{z}$ (EI): $440\left[\mathrm{M}^{+}\right](22)$.

\section{Reaction of 15 with Lawesson's reagent 1. Preparation of compound 17}

To a stirred solution (11.4 mmol) of ethyl (2E)-2-cyano-3-(4-oxo-4H-chromen-3-yl) acrylate (15) in dry toluene $(25 \mathrm{~mL})$, Lawesson's reagent $1(1.4 \mathrm{~g}, 5.9 \mathrm{mmol})$ was added. The reaction mixture was refluxed for $10 \mathrm{~h}$ until (TLC) and then evaporated under reduced pressure. The solid formed was collected and recrystallized from acetone/ether to give (5Z)-2-(4-methoxyphenyl)-5-[(4thioxo-4H-chromen-3-yl)methylene]-1,3,2-thiazaphosphinane-4,6-dithione-2-sulfide (17) as yellow crystals $\left(69 \%\right.$ yield), m.p $179-180{ }^{\circ} \mathrm{C}$. Found: C, 49.00, H, 2.99, N, 2.59, P, 6.61, S, $32.52 \% \mathrm{C}_{20} \mathrm{H}_{14} \mathrm{NO}_{2} \mathrm{PS}_{5}$ (491.65) requires $\mathrm{C}, 48.86, \mathrm{H}, 2.87, \mathrm{~N}, 2.85, \mathrm{P}, 6.30, \mathrm{~S}, 32.61 \%$; $v_{\max }(\mathrm{KBr}) / \mathrm{cm}^{-1}: 3180(\mathrm{NH}), 1570(\mathrm{C}=\mathrm{C}), 1180(\mathrm{C}=\mathrm{S}), 1130(\mathrm{~S}=\mathrm{C}-\mathrm{NH}), 1070(\mathrm{~S}-\mathrm{C}=\mathrm{S}), 680$ $(\mathrm{P}=\mathrm{S}) ; \delta_{H}(\mathrm{ppm}): 3.82\left(\mathrm{~s}, 3 \mathrm{H}, \mathrm{OCH}_{3}\right), 6.8(\mathrm{~s}, 1 \mathrm{H}$, exocyclic ethylenic proton), 7.06, $8.08(2 \mathrm{dd}, 4 \mathrm{H}$, $\left.J_{H H}=9 \mathrm{~Hz},{ }^{3} J_{H P}=10.5 \mathrm{~Hz}, H-\mathrm{Ar}\right), 7.5,8.74\left(2 \mathrm{~d}, 2 \mathrm{H}, J_{\mathrm{HH}}=7.5 \mathrm{~Hz}, H-\mathrm{Ph}\right), 7.26,7.35(2 \mathrm{t}, 2 \mathrm{H}$, $=\mathrm{CH}-\mathrm{CH}=), 9.54(\mathrm{~s}, 1 \mathrm{H}, \mathrm{CH}-\mathrm{O}), 10.94$ (br., $1 \mathrm{H}, \mathrm{NH}) ; \delta_{C}(\mathrm{ppm}): 54.69\left(\mathrm{OCH}_{3}\right), 84.90,118.55$, $119.89,120.86,124.58,129.85,134.21,138.05,148.15,152.40,157.22,158.22,158.59$ (aromatic carbon atoms), $196.10\left(\mathrm{~d}, \mathrm{~S}=\mathrm{C}-\mathrm{NH},{ }^{2} J_{C P}=34 \mathrm{~Hz}\right), 201.16(-\mathrm{C}=\mathrm{S}$, ring), $221.56(\mathrm{~d}$, $\left.\mathrm{S}=C-\mathrm{S},{ }^{2} J_{C P}=35 \mathrm{~Hz}\right)$; $\delta_{P}\left(\mathrm{CDCL}_{3}\right): 104.8 \mathrm{ppm} ; \mathrm{m} / \mathrm{z}(\mathrm{EI}): 491\left[\mathrm{M}^{+}\right](28)$.

\section{Acknowledgements}

We are grateful to Dr. Latifa A. Mohamed, Division of Genetic Engineering and Biotechnology, Department of Microbial Chemistry, National Research Centre, for the pharmacological evaluation.

\section{References}

1. (a) Scheibye, S.; Shabana, R.; Lawesson, S.-O.; Romming, C. Tetrahedron 1982, 38, 993. (b) Ozturk, T.; Ertas, E.; Mert, O. Chem. Rev. 2007, 107, 5210.

2. Cava, M. P.; Levinson, M. I. Tetrahedron 1985, 41, 5061.

3. Brillon, D. Sulfur Rep. 1992, 12, 297.

4. Apple, R.; Knoch, F.; Kunze, H. Angew. Chem. 1983, 95, 1008.

5. Ecker, A.; Boie, I.; Schmidt, U. Monatsh. Chem. 1973, 104, 503.

6. Clausen, K.; Pedersen, B. S.; Scheibye, S.; Lawesson, S.-O.; Bowie, J. H. Int. J. Mass Spectrum Ion Phys. 1979, 29, 223.

7. Clausen, K.; Lawesson, S.-O. Nouv. J. Chim. 1980, 4, 43.

8. Shabana, R.; Osman, F. H.; Atress, S. S. Tetrahedron, 1993, 49, 1271.

9. Khidre, M. D.; Yakout, E. S.; Mahran, M. R. Phosphorus Sulfur Silicon 1998 133, 119. 
10. Crutchfield, M. M.; Dungan, O. H.; Letches, J. H.; Mark, V.; van Wazer, J. R. Top. Phosphorus Chem. Interscience Puplishers, a Division of John Wiley and Sons. 1967, Vol. 5, Chap. 4, pp 373, 374.

11. Shabana, R.; Mahran M. R.; Hafez, T. S. Phosphorus Sulfur Silicon 1987 31, 1.

12. Bigoli, F.; Pellinghelli, M. A.; Atzei, D.; Deplano, P.; Trogu, E. F. Phosphorus Sulfur Silicon 1988, 37, 189.

13. Arca, M.; Cornia, A.; Devillenova, F. A.; Fabretti, A. C.; Isaia, F.; Lippolis, V.; Verani, G. Inorg. Chim. Acta 1997, 262, 81.

14. (a) Abass, M. Phosphorus, Sulfur, Silicon Relat. Elem. 2003, 178, 1413. (b) Testa, M. G.; Perrini, G.; Chiacchio, U.; Corsaro, A. Phosphorus, Sulfur Silicon Relat. Elem. 1994, 86, 75.

15. (a) Pederesen, B. S.; Lawesson, S.-O. Tetrahedron 1979, 35, 2433. (b) Golovko, T. V.; Soloveva, N. P.; Granik, V. G. Mendeleev Commun. 1995, 191.

16. Moustafa, H. M. Phosphorus Sulfur Silicon 2000, 164, 11.

17. Pederesen, P. S.; Scheibye, S.; Nilson, N. H.; Lawesson, S.-O. Bull. Chem. Soc. Belg. 1978, 87, 223.

18. Scheibye, S.; Pedersen, P. S.; Lawesson, S.-O. Bull. Chem. Soc. Belg. 1978, 87, 229.

19. Pederesen, P. S.; Scheibye, S.; Clausen, K.; Lawesson, S.-O. Bull. Chem. Soc. Belg. 1978, 87, 293.

20. Fitton, A. O.; Frost, J. R.; Houghton, P. G. J. Chem. Soc., Perkin Trans. 1 1977, 1450. 